\title{
Early warning information system for land degradation hazards in New Suez Canal region, Egypt
}

\author{
El-Sayed E. Omran ${ }^{1}$
}

Received: 30 May 2016/Accepted: 4 June 2016/Published online: 15 June 2016

(C) Springer International Publishing Switzerland 2016

\begin{abstract}
Advancement in technology and communications has given way to exceptionally refined early warning systems for environmental monitoring. Three hazards are recognized in New Suez Canal region in which water table is the most imperative one. Early warning information systems (EWISs) have proven to be a decent tool for monitoring most environmental hazards. A prototype of actual near real-time EWIS for water table rising management was executed using various sensors. A system of remote sensors would quantify water level hazard in nearby wells which would enroll when the water level increment over a pre-identified level. The sensor would send this information back to the server. The server would recognize when a critical number of wells expanded over the preidentified level, indicating an imminent challenge to the water table. When water level increase above a critical level, the sensor would share this information with a centralized monitoring server. At this point, the data is handled inside the geographical information systems. Subsequently, the early warning panel would be up hosted to the website, whereby such information would be made accessible to the public and authorities involved. An alarm would be issued to relevant authorities, triggering and enabling a water management response. The results of assessment analysis proved that there are a lot of resources that could utilize better future opportunities. There is additionally some threats in the future that may restrain its improvement process. Many obstacles exist in efforts at establishing EWISs and most need to be addressed to enhance the
\end{abstract}

El-Sayed E. Omran

ee.omran@gmail.com

1 Soil and Water Department, Faculty of Agriculture, Suez Canal University, Ismailia 41522, Egypt creation of completely integrated and effectively operational EWISs.

Keywords Water table $\cdot$ Water management $\cdot$ Early warning · Assessment analysis - New Suez Canal - Egypt

\section{Introduction}

Land degradation is an increasing issue globally, exacerbated by environmental change and influencing food security, threatening water resources and eventually influencing various soil threat issues (Gleditsch 2012). Three critical land degradation threats are recognized in Suez Canal Region, Egypt (Omran 2016) which affect soil and water management: water table rising; desertification; and salinization. The New Suez Canal (NSC) region has 'double trouble' range of impacts, at nearby and territorial scales, on both the ecological and environment of the territory. One of these impacts is a water-logged area. Waterlogged area which is an environmental issue is found throughout the world (Chowdary et al. 2008; Minar et al. 2013; Quan et al. 2010; Qureshi et al. 2008) and Egypt (Omran 2012a, b, 2016). These threats are bringing on significant issues influencing a huge number of hectares of land either directly or indirectly, not only affecting the soil but also leaving the soil to cause contamination to water bodies. Governments and communities have to actively react to these threats aiming at minimizing the rate of this change and raising the resilience of local communities to cope with its a devastating impact on socio-economic development. The NSC region has been facing drastic water table rising which the traditional methods to mitigate it are cost and time consuming. There is, therefore, the need to monitor and early predict such trends to help 
reduce associated impacts. Monitoring and predicting these threats and issuing timely warning systems are crucial to mitigate their disastrous impact on population, environment and economy (Ashraf et al. 2014).

The early warning system (EWS) technique is aimed at tracking changes as 'early' as possible. Early warning depicted the process of detecting a possible threat using prediction modeling and experts and warning crisis managers and decision makers (Grasso 2005, 2012). These models utilize the real circumstances and predictions which results in expected threats, with a margin of uncertainty (Jonkman 2007; van Noortwijk and Barendregt 2004). When these predictions surpass predefined warning or safety level, alarms will be triggered (Ministry of Transport 2008; Kolen et al. 2009) and crisis organizations will be put into place. Early warnings permit the organization to adapt early, forward-thinking change strategies, in order to gain competitive advantages.

To the best of my knowledge, there exist no operational early warning systems to be implemented in the NSC region. There still exist knowledge gaps to interpret comprehensively acknowledged standards on EWSs into action-oriented modalities. The main objective of this study is to propose, build, and implement an operational early warning information system (EWIS) in NSC region, Egypt. To accomplish the main objective, the following specific research objectives are recognized to:

1. Design and build a holistic framework for land degradation warning system based on wireless sensors;

2. Setup an operational EWIS for water table monitoring and management; and

3. Assess the proposed EWIS using situation analysis.

\section{Fundamental requirements to design an early warning system}

This study aims to advance systemic change for a more integrated and holistic approach to mitigate land degradation, through providing inputs to develop EWIS in the NSC region, Egypt. Early warning systems are the integration of four main elements (Villagran de Leon et al. 2006): risk knowledge and risk assessments which give key data to set needs both for mitigation and prevention strategies; monitoring and predicting which provide timely estimates of the potential risk faced by communities, economies and the environment; dissemination and communication systems which are required for delivering warning messages (reliable and simple) to the potentially affected communities; and response where coordination, good governance and appropriate action plans are key points in viable early warning. The basic idea that administers early warning is that the earlier and the more accurately it is possible to predict short and long-term risks, the more likely disasters' impact on society, economies, and environment will be managed and mitigated (UNEP 2012). The configuration of a warning system relies upon the specific elements of the hazard phenomenon, the vulnerability of the region, and the requirements of the potentially affected communities. When all is said in done the configuration of a warning system must provide the following fundamental requirements (Zillman 2003):

1. A sound scientific basis of understanding of the environmental phenomenon concerned.

2. Rapid access to worldwide data streams in order to initialize prediction models for the short- to mediumrange prediction process.

3. Rapid access to regional data, including locally processed remotely sensed data, in order to initialize short-range regional models and mesoscale models to provide prior and during-the-event guidance on the fine time and space scales, and also to provide monitoring of conditions during the event;

4. Hardware and software to store and retrieve data, to examine and display information, and to perform the extensive calculations required for prediction models.

5. Systems to automatically detect and alert forecasters to the likelihood of severe events based on observational or prediction data.

6. Communications to provide information to emergency management authorities and other users with lead times that allow adequate response time for emergency managers to complete preparedness action.

\section{Materials and methods}

\section{Study area}

The study area, Suez Canal region, (Fig. 1) is located at the northern part of Egypt. It is geographically located between $31^{\circ} 44^{\prime} 16.7^{\prime \prime}-32^{\circ} 53^{\prime} 24.1^{\prime \prime} \mathrm{E}$ and $30^{\circ} 02^{\prime} 11.6^{\prime \prime}-31^{\circ} 10^{\prime} 18.5^{\prime \prime} \mathrm{N}$. The soils of the study area are almost sandy, deep soil profile and characterized by clay to clay loam at some sites. Many sabakhas with shallow to moderately shallow water table are scattered in the area. The area (Fig. 1) is covered by Quaternary deposits with variable thickness (Geriesh et al. 2014). The region is influenced by of arid condition (Shata 1998). Arid climate prevails over the whole area at least since the Pleistocene through Mioscene times and the natural vegetation cover is very low (Abdel Samie et al. 1982). Therefore, the remaining effective soil-forming factors which had an active role to be considered in soil development are; topography, parent material and time. 


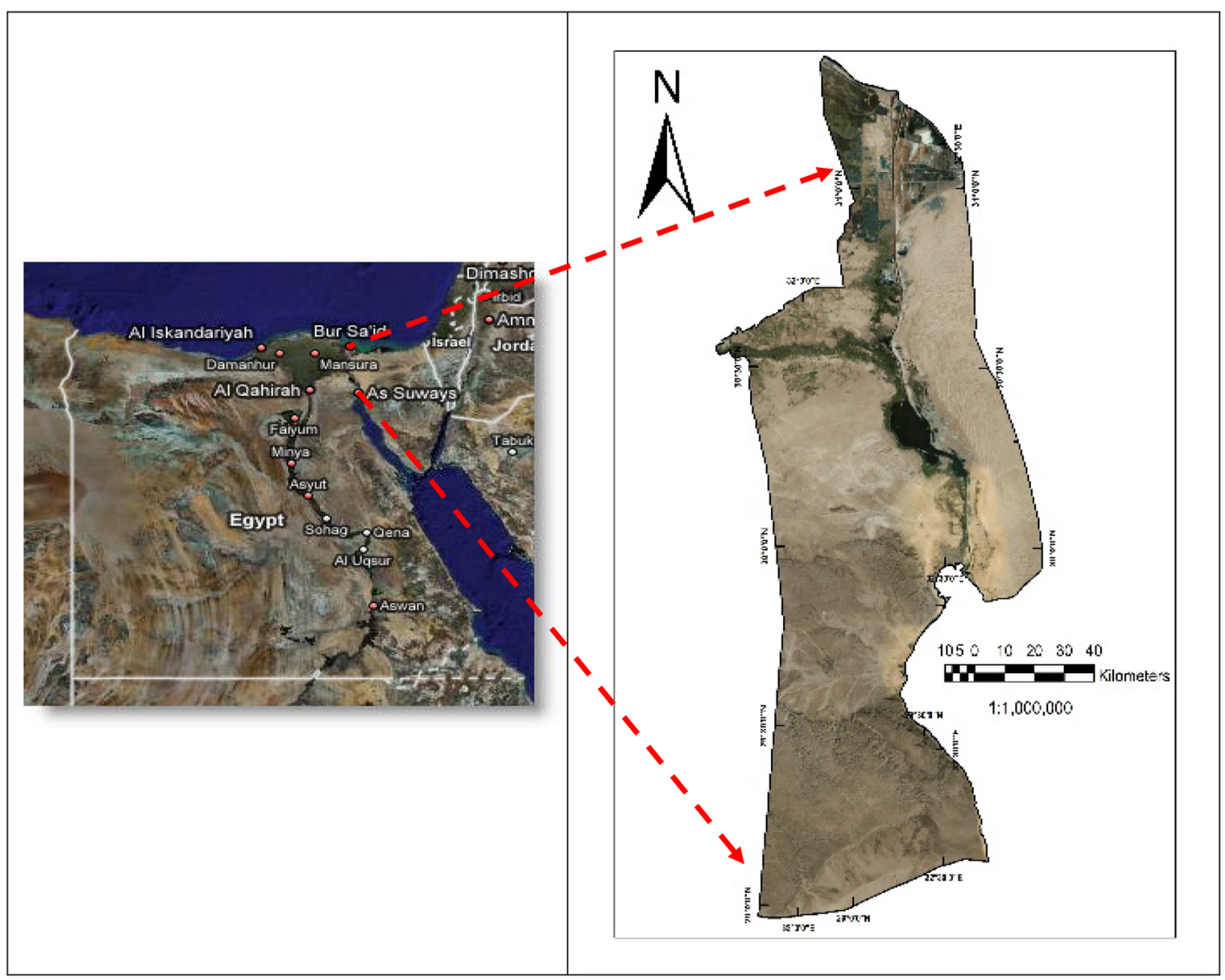

Fig. 1 Location map of the study area

\section{An EWIS framework for land degradation prediction}

Early warning information system (EWIS) proposed in this study characterized as "the provision of timely and effective information, through identified warning system, that permits individuals, communities and businesses exposed to a hazard to take action to dodge or reduce their risk and prepare for effective response". Warnings must get the message across and stimulate those at risk to take action. Five general components to effective early warning information systems may be distinguished conceptually, though in practice these elements are closely inter-related. Nevertheless, these components provide a useful logical framework against which to assess early warning capacities.

- Risk, hazard and vulnerability assessment analysis, which incorporate mapping areas likely to be influenced by the hazards. Risk assessment applies estimates of hazard and plays a vital role in identifying which areas are most vulnerable to hazard, thus prioritizing actions.
- Hazard detection and (warning) prediction. Accurate prediction and timely warnings for disasters can significantly decrease their threats. Remote sensing technology has revolutionized the detection of hazards, which have added precision to the tracking, monitoring, and determine the spatial extent hazard intensity. Clearinghouse mechanism, Oracle, SQL-Server are used to access the data and database management. However, modules and GIS software used to monitor and analysis the data.

- Formulation of warning messages after the hazard has been detected or predicted. An effective hazard-warning message ought to contain the time of detection, location, level and speed of movement, associated risk and the general area at risk. Risk and vulnerability analysis, which include spatial distribution maps of hazards are pre-request before formulation of warning messages.

- Dissemination of warning messages to the target public, authorities, and neighborhood level disaster managers require a communication system with a wide reach. Delivering and exchange of information to emergency 
management authorities are done through Web-based, mailing lists, data bases on line, bulletins, and reports.

- Community (awareness) response to respond to warning in a fitting way is the greatest challenge faced by disaster managers. The vulnerability maps that are regularly updated serve as critical input in effective response to warning.

EWIS for land degradation is focused on producing risk/vulnerability maps and early warnings on potential trends in these threats. Acquisition of baseline data for the EWIS setup such as current land-use/cover, topographical map, soil and rainfall information within the study area is important. Such information would then be used for simulation and modeling of data for input into the EWIS. It is critical that spatial and non-spatial data on all affected areas is captured at the same specific time in a very systematic and reliable way. Earth observation (EO) technologies are utilized to collect data for EWISs. The use of remotely sensing data (e.g., radar (SAR), hyper-spectral sensors, and multiple sensors) allows for the combination of different spatial, temporal, spectral, and radiometric resolutions to (1) identify trends and locate vulnerable areas (small-scale monitoring), and (2) assess vulnerability and predict possible scenarios (large-scale mapping) (Holecz et al. 2003).

A standout amongst the most essential EWIS components is partnerships within and between institutions involved in EWIS. The responsibility for development and maintenance of the data set (data provider) needs to reside with a particular agency or organization, the data custodian. It is important to identify the custodians of base data for EWIS, together with some basic indicators relating to data type and data access practices. The goal of data access is to ensure the quality and availability of data and related information. The Clearinghouse services center is a distributed architectural design where metadata search and geo-processing services are conducted (Omran 2005). Upon entering the electronic front door of the geospatial data one-stop shop, a range of services and information is presented to the user. Upon selection of the specific service, the request processor/administrator will analyze and send the request to the appropriate sector node. The request will be handled from the neighborhood server and the results packaged sent to the electronic front door where a brief description of the data is presented to the user. In the event that the client is fulfilled and acknowledges the outcomes a more complete metadata result will be presented along with recommendations on use and information regarding distribution and delivery.

\section{Assessment analysis for the proposed EWIS in Egypt}

SWOT (strengths, weaknesses, opportunities, and threats) analysis has been utilized to assess the current situation and the environmental factors influencing the organization. Assessment of current status and the external environment is the initial phase in the EWIS process. Based on the interview and literature, information for the SWOT analysis was obtained. Scanning the internal environment for EWIS application in terms of strengths and weaknesses will help to utilize the resources with optimum usage. Scanning the external environment in terms of opportunities and threats, will help to identify the political, economic, social and technological forces (PEST analysis) they deal with, to establish future strategies. Changes in the external environment present new opportunities and new ways to reach the objectives (goals). So, we should select the opportunities that it can pursue with a higher probability of success.

\section{Results}

\section{Land degradation prediction using a wireless sensor network}

Figure 2 demonstrates the wireless sensor network, WiFi and sensor node utilized for land degradation prediction. Different wireless sensors can be used for sensing different land degradation hazards especially related to salinization, water table rising, erosion, drought and desertification. The sensor will utilize the signals, which needs to be transmitted to the users. Transmitting the electrical signal from the gadget in the field to the users through wire is not practical, since it is so long and we have many wells and sensors at various locations in the field. Utilization of commonly used wireless technology is also not advisable due to complexity of the communication system and the powerful needs of such systems. Demonstrating power supply or having a solar panel is also not economical. High battery power may be needed by such devices and regular charging or replacement of the batteries is highly costly. Alternative solution is to provide power from a solar panel. The solar panels are of high cost and hence are after prove to theft. A wireless sensor network (WSN) can be considered to monitor land degradation hazards and to cooperatively pass their data through the network to a main location.

From an implementation point of view sensor nodes are set in the study area under the control of WSN. The base station is stationary and it gathers the data from sensor nodes. The controller performs tasks, processes data and controls the functionality of other components in the sensor 


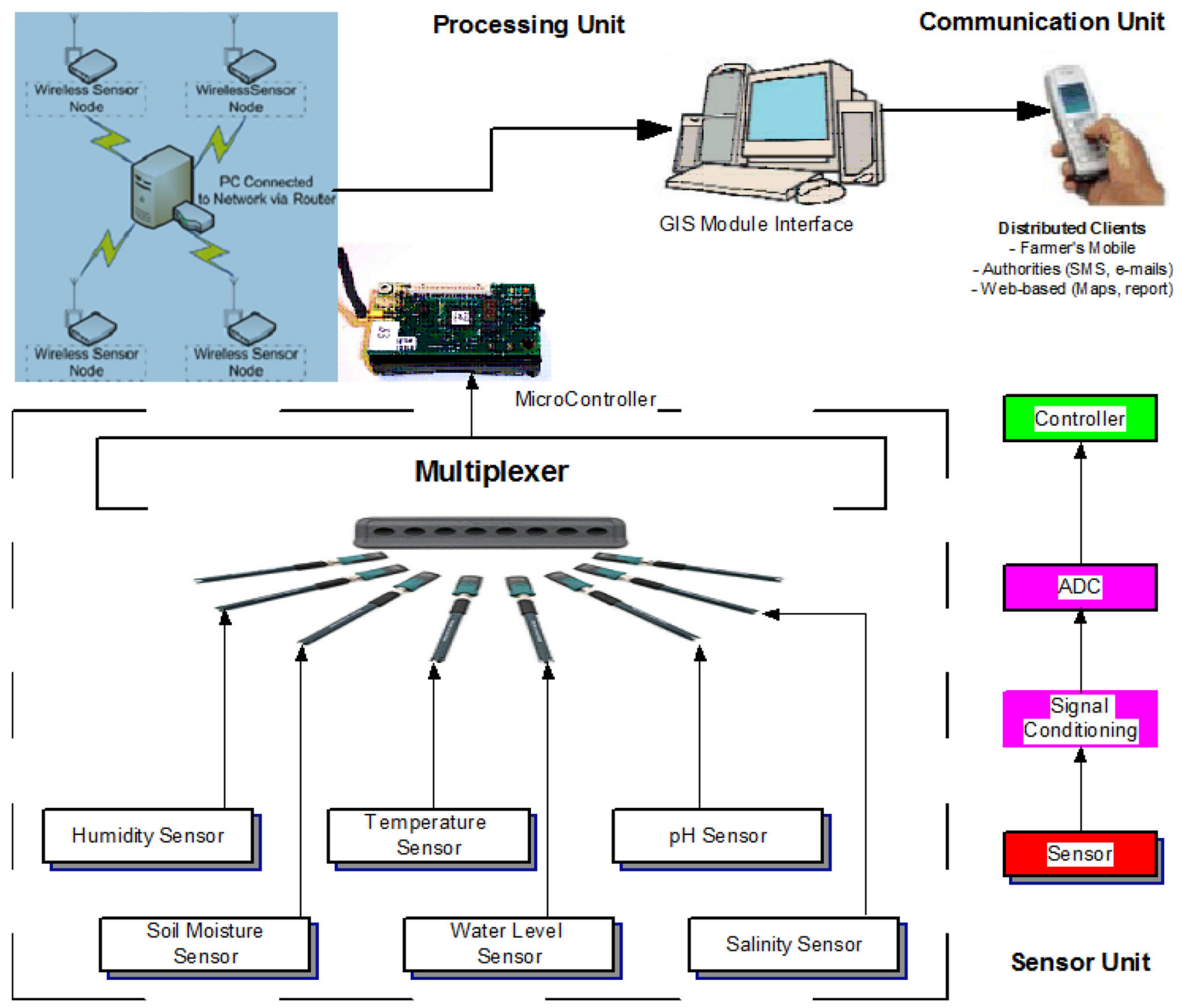

Fig. 2 Concept of wireless sensor network, WiFi and sensor node for land degradation prediction

node. Sensors are hardware devices that produce a quantifiable response to a change in a physical condition such as water level, soil moisture, temperature or pressure (Fig. 2). The consistent simple sign created by the sensors is digitized by an analog-to-digital converter and sent to controllers for further handling. A sensor node should be small in size, consume extremely low energy, operate in high volumetric densities, be autonomous and operate unattended, and be adaptive to the environment. Various wireless standards have been established. Among them, the standards for wireless LAN, IEEE 802.11b ("WiFi") (IEEE 1999b) and wireless PAN, IEEE 802.15.1 (Bluetooth) (IEEE 2002) and IEEE 802.15.4 (ZigBee) (IEEE 2003), are used more widely for measurement and automation applications. An 802.11 (WiFi) mesh network comprised of high-end nodes, such as gateway units (Fig. 2).

This system can be utilized for the early detection, monitoring and prediction of soil salinization, desertification, and water content for agricultural automation. The system depends on satellite-derived data for the near real-time monitoring of vegetation indexes that allows the early detection of vegetation stress and conditions in vegetation health and productivity. The monitoring of phonological parameters allows the assessment of the current vegetation productivity and its projection toward the end of the growing season (Bellone et al. 2009). 


\section{Implementation of the near real-time EWIS for water table rising prediction}

Could we install a system of remote sensors in local (water) wells in order to measure and oversee water table levels? Once the data is completed, a prototype of actual real time outdoor transmission unit is then developed using different sensors (Fig. 3). The main objective of the EWIS is to measure the water table level data from the observation wells and forward it to the central main system for further processing. A system of remote sensors would quantify water level hazard in neighborhood wells. This would recognize the relevant wells in the given water table (Fig. 3), and install four sensors in every well which would register when the water level increase above a preidentified level (at depths 50, 75, 100, $150 \mathrm{~cm}$ ). Water wells can be made according to the need and the water level in each well can be monitored. These observation wells where installed for recording and monitoring the fluctuation of water table. We have taken about $40 \mathrm{~mm}$ PVC pipes each of length $1.5 \mathrm{~m}$, there is $5 \mathrm{~mm}$, holes for $10 \mathrm{~cm}$. Each head nodes control these wells. The wells are set as shown in Fig. 4.

The sensor will create the signals when contact with water. The sensor would send this information back to the server. The server would recognize when a critical number of wells increased over the pre-identified level, indicating an imminent challenge to the water table. When water level increase above a critical (a certain threshold) level, the sensor (Fig. 3) would share this information with a centralized monitoring server. A SIM-card based GPRS transmitter, which is attached to the interface module of the Sensing and Transmission Unit (RSTU) would then convey messages/web record exchanges at consistently interim, so that the data could be transferred to a receiving unit (a server connected too fast Internet line).

At this point, the e-mail/transferred file is read and data is processed within the geographical information systems (GIS) (Fig. 4). The last processed values would then be set against the threshold values, which will trigger an early warning if any of the values exceeds the threshold limits. Subsequently, the early warning panel would be up hosted to the website (http://cms.iti-ismailia.com), whereby such information would be made accessible to the public and authorities involved (Fig. 5). An alert would be issued to relevant authorities, triggering and enabling a water management response. The website's map of different risk areas will be updated as data from the sensors are constantly calculated. A blinking red light with a continuous playback of the 'warning' sound and flash light would

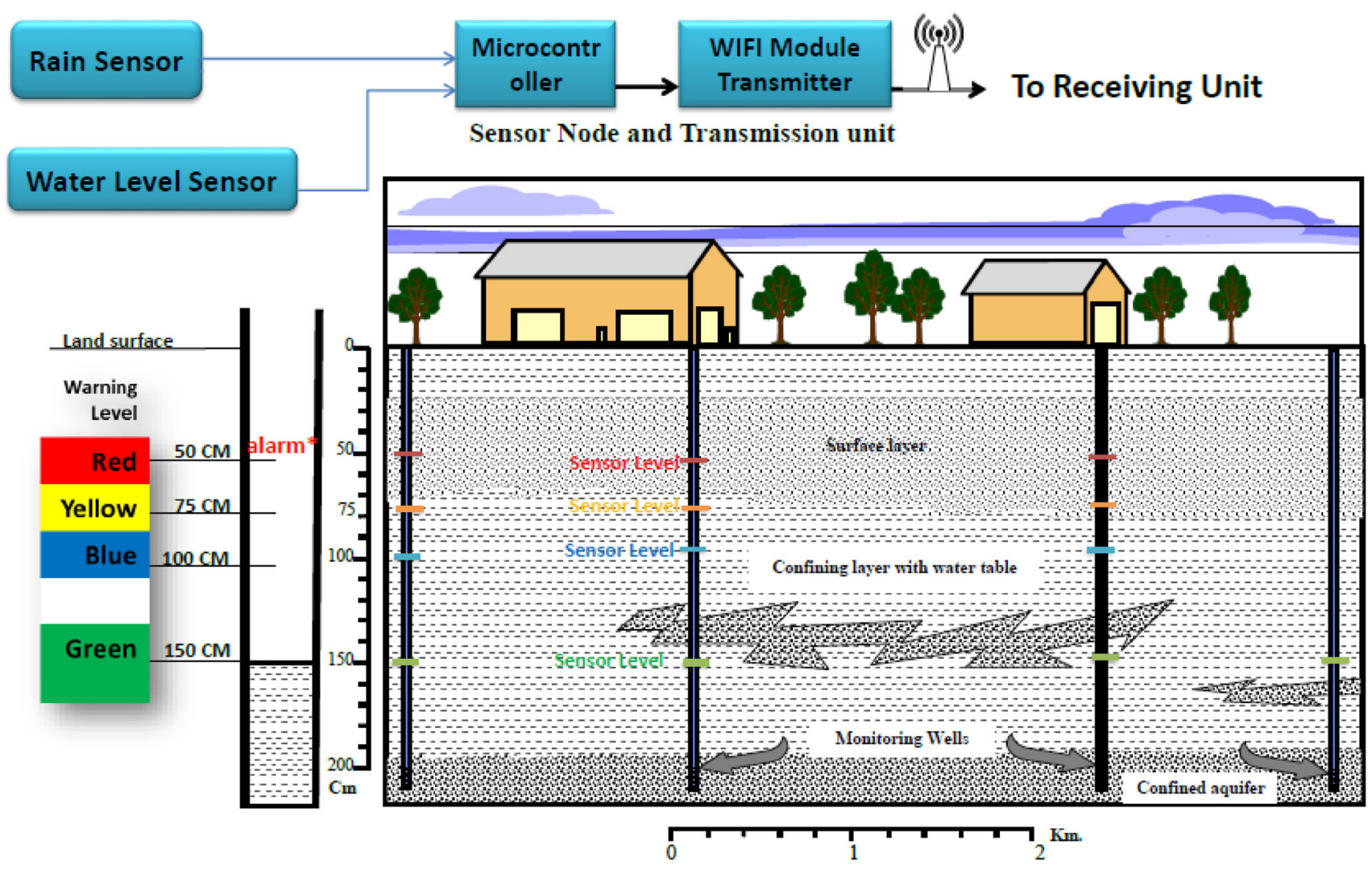

Fig. 3 Flowchart of real time outdoor transmission unit and sensors for water table warning levels showing location and depths of wells 


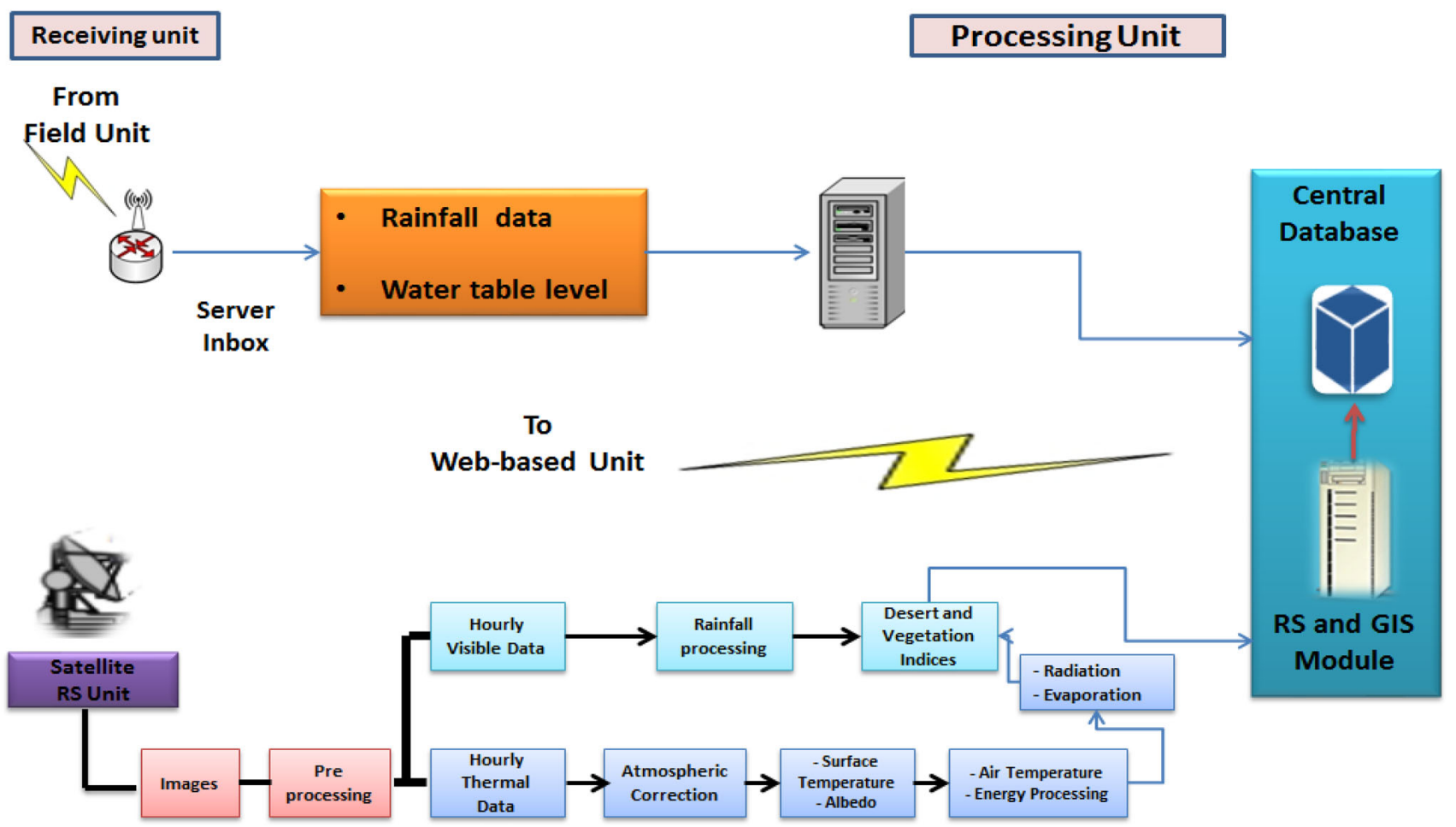

Fig. 4 Receiving unit, administrative unit, server, and control database unit linked with remote sensing and GIS environment

indicate that a certain area is at high risk while yellow is for medium and blue for low risk locations. Remarks on the percentage of risk area and in acreage for the built-up areas, roads and agricultural areas would also be appeared in the display panel.

Figure 6 shows the actual real time implementation of EWIS prototype. The operational system collects all data and coordinate computations required to create the different hazard maps. These incorporates observations of water level and salinity. In daily operations, the EWIS system will be used for management. Automated runs are scheduled daily, taking after the arrival of new data and predictions. Model output can be inspected and compared with observations, to assist forecasters to decide on appropriate warning levels. The distributed water level warnings are based on model predictions and on expert judgment of the forecasters. EWIS has powerful graphical tools for viewing time series data; point time series collection; and editing capabilities-copy to-from e.g., Excel. All models, including data-assimilation, are scheduled to run automatically.

\section{What current conditions support further EWIS improvements?}

The results to establish the improvement goals for the EWIS, according to the internal and the external scanning for the organization, are shown in the SWOT matrix
(Table 1). The results of SWOT analysis demonstrated that there are considerable resources that could utilize better future opportunities. There is also some threats in the future that may restrain its improvement process. The future improvement actions of the EWIS should be identified according to this situation analysis. The strategies that identified based on the problem analysis part and based on the interview, are presented in Table 1. Establishing the improvement actions will definitely impact the external threats and the internal problems (weaknesses) of the system. Developing these actions has to be within the available resources, so that the derived system can fit with the current situation. One of the strategies distinguished in SWOT matrix is focus on a couple of reachable objectives based on the current situation (S2-T1). So, according to this, all the 23 actions identified in SWOT matrix (Table 1) were brought down to 5 future improvement based on the outcomes recognized in the problem analysis part; interview results, user requirements analysis; and SWOT analysis matrix.

First is EWIS awareness creation, which include: promotion of EWIS principles through workshops, training courses and material; provide "train-the-trainer" technical workshops to explain the origins, purpose, and strategies for implementation of the EWIS; and facilitate information sharing through newsletters, web pages, and publications. The introduction of new technology into any 


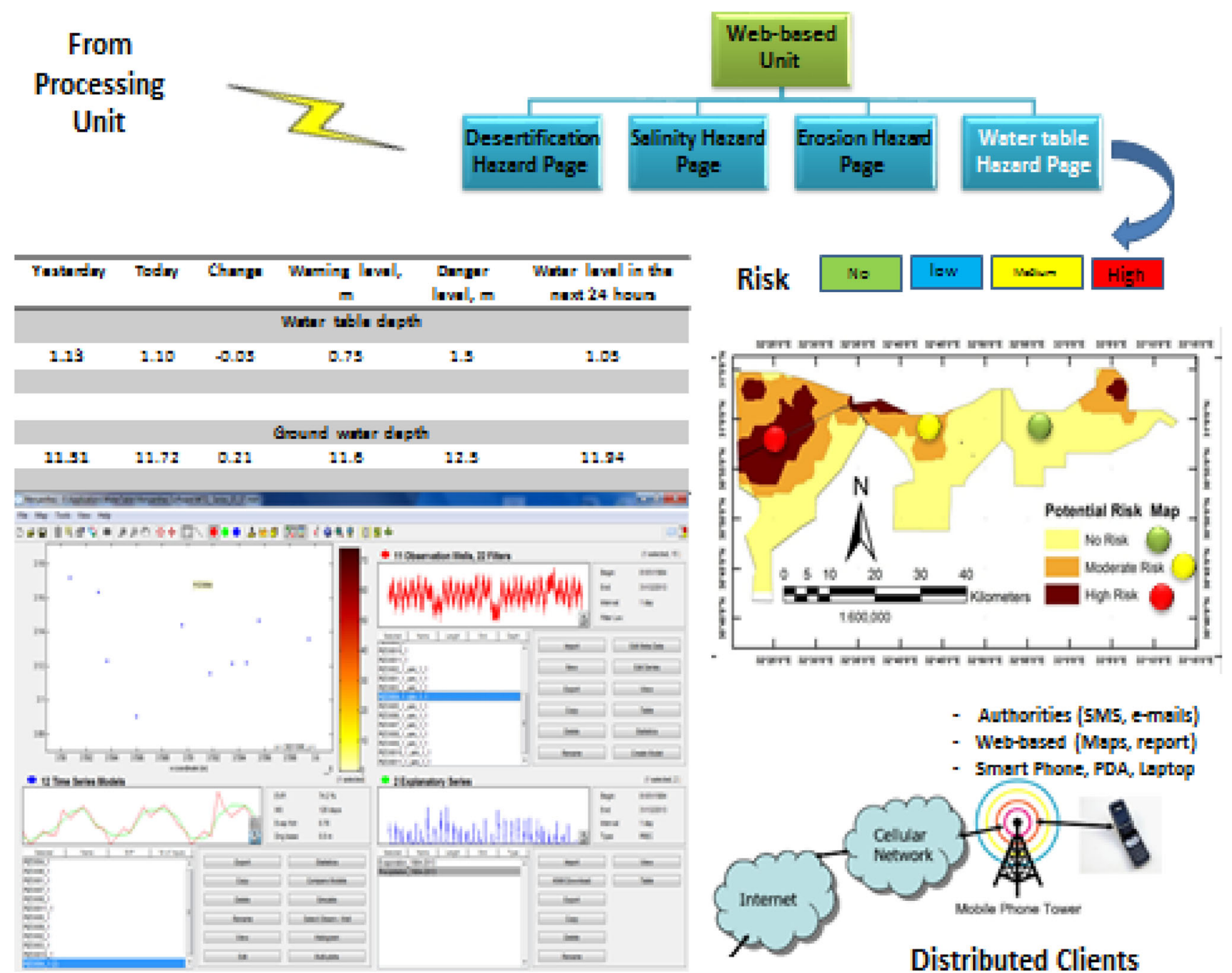

Fig. 5 Screen shot of the operational EWIS shows sample of daily report on water table level, modeling and simulation results of water table depth and different hazard levels and Website ready for access from different clients

organization usually involves retraining of existing staff. Lack of man power with the necessary background is severe. Second is execution of EWIS policy and procedure to facilitate spatial data exchange. All the necessary arrangements that should be made in order to make spatial data and associated services really available and EWIS really work are called institutional framework. Third is establish of EWIS coordination unit at higher policy and executive levels. The organizational strategy should incorporate different levels ranging from the higher policy formulating body to the implementation level. For effecting improvement on the issue under consideration, the following bodies are $\mathrm{P}$ aramount: executive committee, spatial information board, secretariat, and working groups.

Fourth is creation of metadata standard, which is crucial in data dictionaries, reference system, metadata quality and data exchange. Metadata standard is a formalized set of properties that describe the characteristics of the contents of datasets. Metadata is an essential requirement for locating and evaluating available data. The core metadata for EWIS datasets should contain, title and description of the data set, date of data set creation and the update cycle, data set originator or creator and supplier, the geographical extent of the data set based on lat./long. coordinates, geographical names or administrative areas, how to order the data set, available formats, and access constraints etc. (Omran 2005). Last is provide tools to search and access the metadata. Set up Clearinghouses or geo-portals by creating spatial data catalogues in the EWIS node agencies and enter the certified metadata of data producers. Spatial data and information are shared, then the datasets are accessible to the EWIS users. The clearinghouse enables users to find, evaluate, and access geographic data through the Internet. 


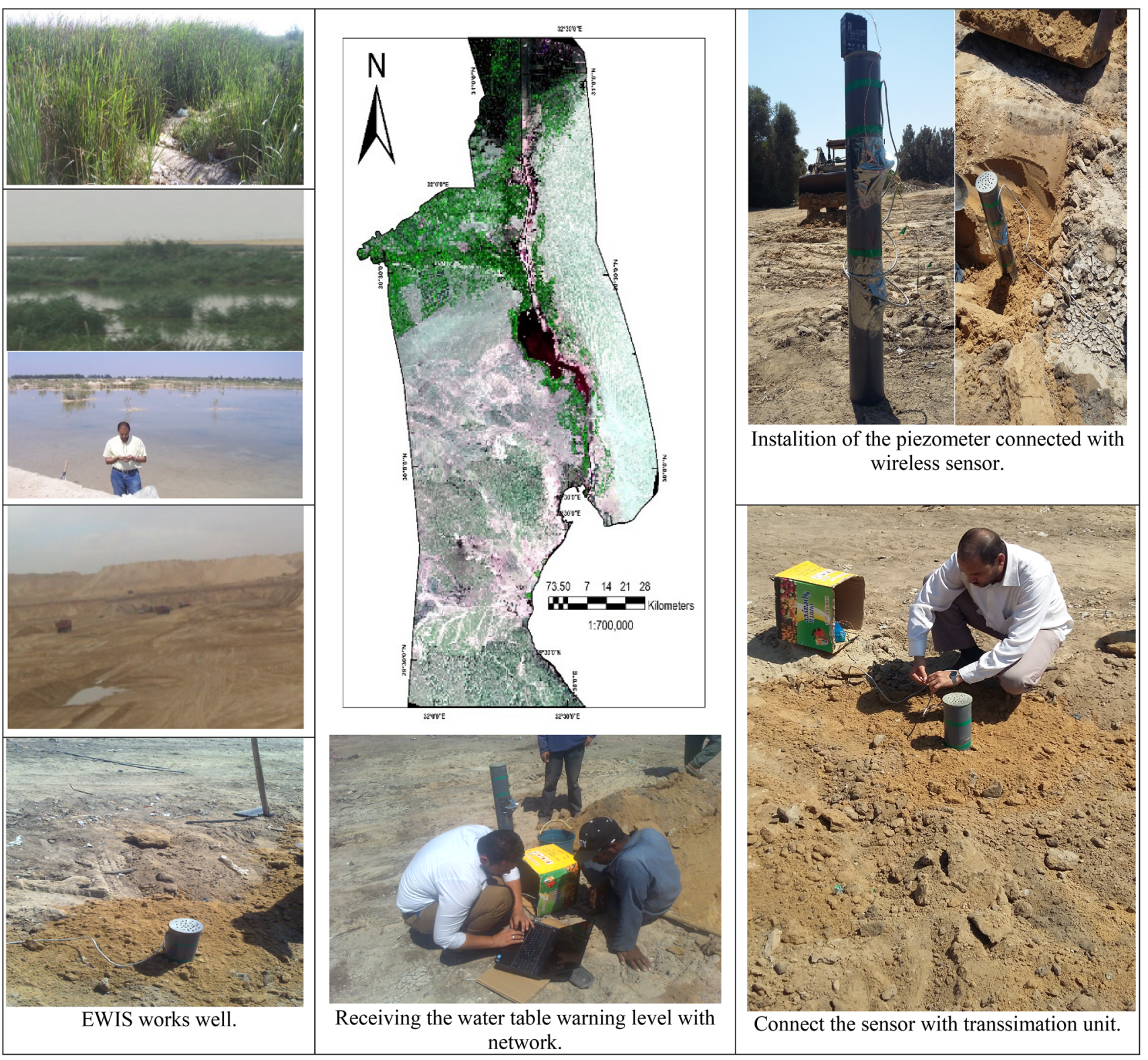

Fig. 6 Actual real time implementation of EWIS prototype and field photos along the Suez Canal region

For the successful achievement of the proposed EWIS, critical success factors (CSF) analysis need to be considered. In order to make the required CSF, the logical base is the bottlenecks identified in the problem analysis part. CSFs determine the projected deficiency in EWIS performance. The idea is simple: in Organization certain factors will be critical to the success of EWIS. If objectives associated with these factors are not achieved, the EWIS will fail. To realize the advantages of EWIS, the following success factors were identified important and crucial for the development of EWIS: partnerships and leadership, data and metadata availability, institutional framework, political support, funding support, capacity building, same standards, and access mechanism.

\section{Discussion}

It is widely realized that the development and implementation of whole EWIS is not an easy task. Developing EWIS requires considerable time, cost, and CSF. Setup and implement full operational EWIS in Egypt will take long time, which is ongoing process. The success of the EWIS depends on continuous efforts of all participants and increasing accumulation systematically. Furthermore, to 
Table 1 SWOT analysis matrix for early warning information system (EWIS) development

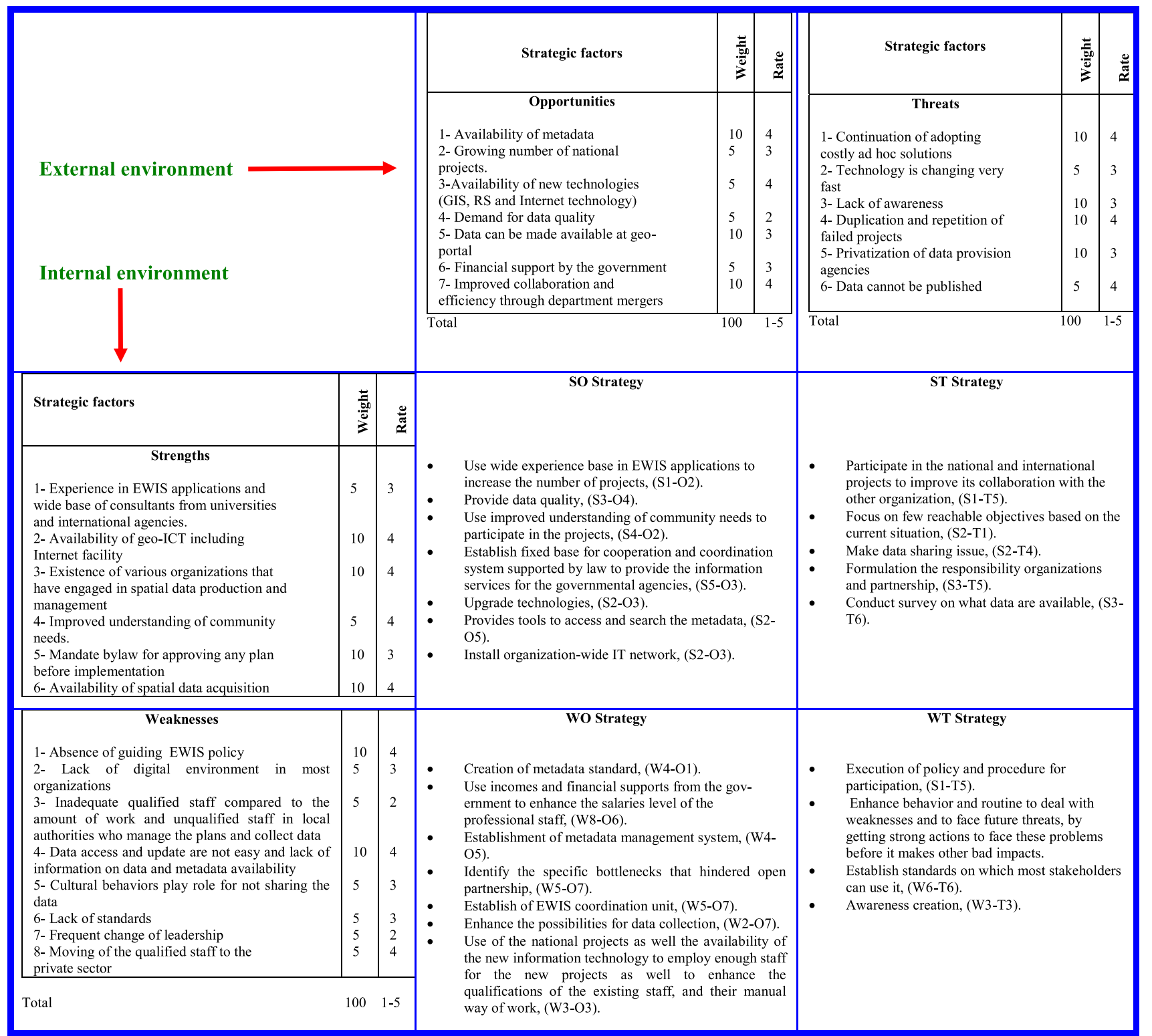

$S$ strengths, $W$ weaknesses, $O$ opportunities, $T$ threats

establish the EWIS, requires huge amounts of money. Setting up a complete EWIS from scratch costs a lot of money. Therefore, in order to utilize all resources, implementation of EWIS prototype in the present study gains more importance.

Successful of the proposed EWIS has contributed immensely to the protection of the environment through the following strengths (advantages). (1) EWIS serve as a vehicle for environmental protection and sustainability. (2) EWIS provide warning information that enable policy- makers, first responders, and personnel to plan and implement emergency response programs. (3) EWIS facilitate decision support systems, providing organizations important information on potential degradation events and enable the formulation of guidelines to resolving such problems. (4) EWIS help identify the source of adverse degradation events, which is important during the restoration of the affected natural resource. (5) EWIS encourage general development and the exploration of scientific capabilities, with great technological advances in sensor 
designs, data analysis, and efficient response plans. (6) EWIS promote institutional combination and joint effort.

In view of current results, spatial information field in Egypt to support EWIS is still immature (weakness) with broad set of issues yet to be resolved. (1) EWIS can be extremely costly, and difficult to establish as automated systems from scratch. (2) $24 \mathrm{~h}$ real-time data collection and transmission are still a challenge. False positive and false negative readings are a source of many unpredicted disasters or misinformation, leading to human fatalities. (3) There is still no best optimal approach to sensor placement that captures potentially all inbound water table levels. (4) Absence of guiding policy. The development of the EWIS is not purely a technical issue, institutional, or other aspects also affect its progress (culture and societal differences). In the present situation, there is no copyright law, and most of the agencies need to market their product in order to find additional resources to maintain and update their data. (5) Data access and update are not easy. It is extremely difficult to access any government data. Existing datasets have been collected to different specification and standards, making it difficult to integrate the data collected from different sources. Most of data-generating agencies do not have the mandate for data dissemination. (6) Coming to the availability and accessibility of spatial data, the situation is worse. The maps are not easily accessible. There does not any system for data accessibility. Digital data are not available with most of the data-producing agencies and even analogue data are not accessible.

Analysis of the SWOT results suggested the following opportunities for the future EWIS development. The first opportunity is enhanced joint effort and coordination. EWIS require a wide range of spatial data, which often come from different data providers and are not compatible. There cannot be a single organization in charge of all the data required for EWIS. That is why the need for coordination between organizations is necessary to achieve the desired goal. The organizational arrangements should be set up as soon as possible to deal with the major issues in establishing the EWIS framework. The spatial information board will be formed at the higher political level (members from parliamentary standing committees, cabinet members) with the main duty of giving directions on the national interest pertaining to Geo-information, make amendments based on the proposals supplied by the Executive Committee. We should also formulate a strategy to develop the EWIS on the top level and start to work on the standards of core data. The second opportunity is the availability of metadata. Existing data has to be organized to form datasets. It must be possible to combine spatial information from different sources and share it between many users and applications. In Egypt quite often, the users without much experience have to specify and find what they exactly need, and they have to fight their way through a maze of officers, rule and regulations, which they barely understand to eventually get what they want. Quite likely, they will give up their project somewhere halfway, or find it cheaper to digitize the data they need. The contrary would be situation where the available datasets are well documented in publish data catalogues, and inexperienced users can get assistance.

Examination of the SWOT results recommended the following threats for the future EWIS advancement: First, reduced international competitiveness. Although the Egyptian users are denied access to any maps, many of the maps are available freely outside Egypt. If Egypt does not make available core data sets, it will lose its competitiveness. Like air, water, and electricity data is also a vital factor for attracting investments. Thus, it is very difficult for Egypt to hide geographic information. We should adopt a pro-active approach regarding spatial data availability instead of being in a reactionary mode. Therefore, we should try to find a number of reasons to share data instead of finding a number of reasons to hide data. Second, continuation of adapting costly ad-hoc solutions. The advantages from spending on geographic data acquisition are low comparable to any other developed nation. Most of GIS activities in Egypt are an ad-hoc undertaking and standalone operational system. This is related to few institutions that have included in acquired GIS. Thirdly, the presence of Egypt in ISO/TC 211 which is international body working in the field of standardization of digital spatial information is nil (Omran 2005).

Five outputs expected from EWIS. First output is the dynamic, long-term planning mechanisms to cope with the environmental change impact, which broaden the national coalition for integrating climate change adaptation into sectoral development. It will likewise develop enhanced understanding of the links between environmental change adaptation and disaster risk reduction, and integration of these into national development plans. Second is the leadership and institutional frameworks to manage climate change in an integrated manner at the local and national levels. This output will result in strengthened functioning inter-ministerial and multi-stakeholder society mechanisms to manage risks and opportunities. The third output is the climate-resilient policies and measures implemented in priority sectors. A gap analysis of EWIS for multiple hazards e.g., drought, desertification, WTD, and salinity will be prioritized. The last output is the knowledge on adjusting national development processes to fully incorporate risks and opportunities generated and shared across all levels. This output will focus on developing platforms for learning-in-action to upscale adaptation efforts and 
create a knowledge base. Actions will support key institutions to document, disseminate, and influence policy for adaptation in priority sectors.

Also there are four expected outcomes. A first outcome is establishment of regulatory framework for ensuring sustainable use of natural resources for improved livelihood. Second outcome is enhance National and local systems for emergency preparedness, disaster prevention, response and mitigation. The third outcome is the importance of application of EWIS is that the earlier and more accurately, we are able to predict potential risks associated with natural and human-induced hazards, the more likely we will be able to manage and mitigate disasters' impact on society, economies, and the environment. The last outcome is the gain awareness of the main current and future threats of climate change and their adverse effects on the region biodiversity.

\section{Conclusions}

Progression in sensor technology and the availability of remotely sensed and GIS data have given way to very sophisticated early warning systems for environmental monitoring. Equally, important components of EWIS are emergency preparedness and disaster response plans that are initiated when an adverse impact is predicted and warning information issued. Before crisis managers act, they must make sense of the possible threat. Sense making is defined as understanding the threat and willingness to think about possible responses. Many obstacles exist in efforts at establishing EWISs and most need to be addressed to enhance the creation of fully integrated and effectively operational EWISs. Issues related to funding, research, expertise, sound technologies, infrastructure, institutional capacity, collaboration and integration, efficiency in response, improved risk management, as well as communication infrastructure, need be looked at holistically in the process of setting up well-integrated EWISs for all potentially dangerous environmental degradation. For the successful achievement of the proposed EWIS, there are different critical success factors that need to be considered. Setup and implement full operational EWIS in Egypt will require considerable time and cost. A prototype implementation of one EWIS unit was presented. Presently, there are at least a good case has been to work for, lay a convenient ground to start improvement, or can see a chance to tweak the situation to make it convenient. The last selection of the best strategy has to be determined on the basis of the government and other stakeholders. This is impossible right now.

\section{References}

Abdel Samie AG, Labib TM, Abdel Hady M (1982) Soil classification and potentials in Sinai Peninsula from Landsat Images. In: International Symposium on Remote Sensing of Environment, Remote Sensing Center, Cairo

Ashraf MA, Maah MJ, Yusoff I (2014) Soil Contamination, Risk Assessment and Remediation. Chapter I INTECH Environmental Risk Assessment of Soil Contamination (3-56). doi:10.5772/ 57287

Bellone T, Boccardo P, Perez F (2009) Investigation of vegetation dynamics using long-term normalized difference vegetation index time-series. Am J Environ Sci 5(4):461-467

Chowdary VM, Vinu Chandran R, Neeti N, Bothale RV, Srivastava YK, Ingle P, Ramakrishnan D, Dutta D, Jeyaram A, Sharma JR, Ravindra S (2008) Assessment of surface and sub-surface waterlogged areas in irrigation command areas of Bihar state using remote sensing and GIS. Agric Water Manag 95:754-766. doi:10.1016/j.agwat.2008.02.009

Geriesh MH, El-Rayes AE, Kaiser MF, Mansour BM, Abd El-Aleem M (2014) Geoenvironmental Impact Assessment of Suez Canal Corridor Development Area, Challenges and Opportunities, Suez Canal and Sinai Province, Egypt. Geology Department, Faculty of Science, Suez Canal University, Ismailia, Egypt

Gleditsch NP (2012) Whither the weather? Climate change and conflict. J Peace Res 49(1):3-9

Grasso V (2005) Seismic early warning systems: procedure for automated decision making. Università degli Studi di Napoli Federico II, p 104

Grasso V (2012) Early warning systems: state-of-art analysis and future directions. Draft report, United Nations Environmental Programme $\mathrm{p} 66$

Holecz F, Heimo C, Moreno J, Goussard J, Fernandez D, Rubio JL (2003) Desertification-a land degradation support service. IEEE 3:1490-1492

IEEE (1999b) Wireless Medium Access Control (MAC) and Physical Layer (PHY) Specifications: Higher-Speed Physical Layer Extension in the $2.4 \mathrm{GHz}$ Band. IEEE Standard 80211b The Institute of Electrical and Electronics Engineers Inc, 345 East 47th Street, New York, USA

IEEE (2002) Wireless Medium Access Control (MAC) and Physical Layer (PHY) Specifications for Wireless Personal Area Networks (WPANs). IEEE Standard 802.15.1. The Institute of Electrical and Electronics Engineers Inc, 345 East 47th Street, New York USA

IEEE (2003) Wireless Medium Access Control (MAC) and Physical Layer (PHY) Specifications for Low-Rate Wireless Personal Area Networks (LR-WPANs). IEEE Standard 802154 The Institute of Electrical and Electronics Engineers Inc, 345 East 47th Street, New York, USA

Jonkman SN (2007) Loss of life estimation in Flood risk assessment. Theory and applications. PhD Thesis Delft University of Technology

Kolen B, Kutschera G, Helsloot I (2009) A comparison between the Netherlands and Germany of evacuation in case of extreme flooding. In COST09 Paris (France)

Minar MH, Hossain B, Shamsuddin MD (2013) Climate change and coastal zone of Bangladesh: vulnerability, resilience and adaptability. Middle East J Sci Res 13(1):114-120. doi:10.5829/idosi. mejsr.2013.13.1.64121

Ministry of Transport PWaWM (2008) Emergency plan "Extreme water level and storm surge". Guideline for a national approach, The Hague 
Omran EE (2005) Spatial Data Infrastructure to Support Land Evaluation Applications in Egypt. MSc Thesis GIRS-2005-016, Centre for Geo-Information, Wageningen Univesity, The Netherlands

Omran EE (2012a) Detection of land-use and surface temperature change at different resolutions. J Geogr Inf Syst 4(3):189-203. doi: $10.4236 /$ jgis. 2012.43024

Omran EE (2012b) A neural network model for mapping and predicting unconventional soils at a regional level. Appl Remote Sens J 2(2):35-44

Omran EE (2016) A stochastic simulation model to early predict susceptible areas to water table level fluctuations in North Sinai, Egypt. Egypt Remote Sens J. doi:10.1016/j.ejps.2016.03.001

Quan RS, Liu M, Lu M, Zhang L, Wang J, Xu S (2010) Waterlogging Risk assessment based on land use/cover change: a case study in Pudong New Area, Shanghai. Environ Earth Sci 61:1113-1121. doi:10.1007/s12665-009-0431-8
Qureshi AS, McCornick PG, Qadir M, Aslam Z (2008) Managing salinity and waterlogging in the Indus Basin of Pakistan. Agric Water Manag 96(1):1-10

Shata A (1998) Potential water resources of the Sinai penensula,a review paper. The National Syposium on Nitrogen-fixing leucaena trees, EI-Arish, Egypt

UNEP (2012) Early Warning Systems: A State of the Art Analysis and Future Directions. In: Grasso, VF, Singh, A (eds) Nairobi. http://www.unep.org

van Noortwijk J, Barendregt A (2004) Available and necessary time for evacuation in case of possible flooding Lelystad: HKV lijn in water

de León JCV, Bogardi J, Dannenmann S, Basher R (2006) Early warning systems in the context of disaster risk management. Entwickl Ländlicher Raum 2:23-25

Zillman JW (2003) Meteorological and hydrological early warning systems. In: Zschau J, Küppers AN (eds) Early Warning systems for natural disaster reduction, Heidelberg, Berlin 